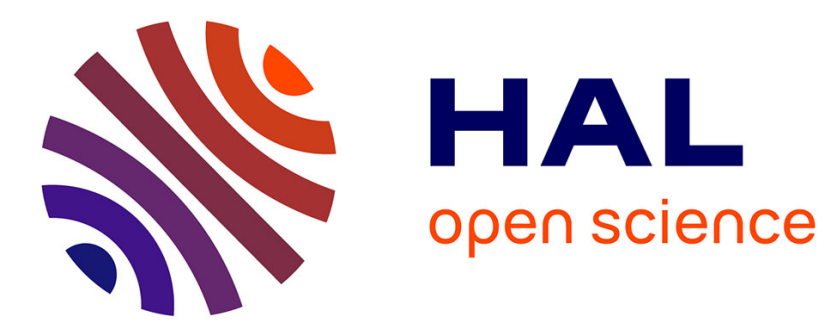

\title{
Predicting the transition from internal to external oxidation of alloys using an extended Wagner model
}

Jean-Baptiste Leblond, Moïse Pignol, Didier Huin

\section{To cite this version:}

Jean-Baptiste Leblond, Moïse Pignol, Didier Huin. Predicting the transition from internal to external oxidation of alloys using an extended Wagner model. Comptes Rendus Mécanique, 2013, 341 (3), pp.314 - 322. 10.1016/j.crme.2013.01.003 . hal-01434008

\section{HAL Id: hal-01434008 \\ https://hal.sorbonne-universite.fr/hal-01434008}

Submitted on 13 Jan 2017

HAL is a multi-disciplinary open access archive for the deposit and dissemination of scientific research documents, whether they are published or not. The documents may come from teaching and research institutions in France or abroad, or from public or private research centers.
L'archive ouverte pluridisciplinaire HAL, est destinée au dépôt et à la diffusion de documents scientifiques de niveau recherche, publiés ou non, émanant des établissements d'enseignement et de recherche français ou étrangers, des laboratoires publics ou privés. 


\title{
Predicting the transition from internal to external oxidation of alloys using an extended Wagner model
}

\author{
Jean-Baptiste Leblond ${ }^{\mathrm{a}}$, Moïse Pignol ${ }^{\mathrm{b}}$, Didier Huin ${ }^{\mathrm{c}}$ \\ ${ }^{a}$ UPMC Univ Paris 6 and CNRS, UMR 7190, Institut Jean Le Rond d'Alembert, F-75005 Paris, France \\ ${ }^{\mathrm{b}}$ ESI Group, Immeuble Le Récamier, 70 rue Robert, 69458 Lyon Cedex 06, France \\ ${ }^{\mathrm{c}}$ ArcelorMittal Global RED, Maizières Automotive Products, 57283 Maizières-lès-Metz Cedex, France
}

\begin{abstract}
Leblond [1] recently estimated the conditions governing the transition from internal to external oxidation of alloys using a variant of Wagner's [2] model incorporating the possible role of oxides as diffusion barriers, through a heuristic dependence of the diffusion coefficient of oxygen upon their local volume fraction. But the crudeness of the formula adopted made the prediction of the onset of external oxidation only qualitative. A more accurate formula is derived here by using a thermal analogy and finite element computations of the reduction of the conductivity generated by nonconducting, more or less flat obstacles. The extended Wagner model incorporating this formula leads to a prediction of the "critical" local fraction of oxides corresponding to the transition from internal to external oxidation, depending on the "aspect ratio" of the oxides. The predicted value is in acceptable agreement with that measured by Rapp [3] for the Ag-In system, for a reasonable postulated value of this aspect ratio.

To cite this article: J.B. Leblond, M. Pignol, D. Huin, C. R. Mécanique? (2013).

Résumé

Prédiction de la transition entre oxydation interne et externe des alliages à partir d'un modèle de Wagner étendu. Leblond [1] a récemment estimé les conditions régissant la transition entre oxydation interne et externe des alliages en utilisant une variante du modèle de Wagner [2] incorporant le rôle possible d'obstacles à la diffusion joué par les oxydes, via une dépendance heuristique du coefficient de diffusion de l'oxygène visà-vis de leur fraction volumique locale. Mais du fait de la grossièreté de la formule adoptée, la prédiction de l'occurrence de l'oxydation externe ne pouvait être que qualitative. On obtient ici une formule plus précise grâce à une analogie thermique et des calculs par éléments finis de la réduction de la conductivité générée par des obstacles non conducteurs plus ou moins aplatis. Le modèle de Wagner étendu incorporant cette formule conduit à une prédiction analytique de la fraction "critique" locale d'oxydes correspondant à la transition entre oxydation interne et externe, dépendant du "facteur de forme" des oxydes. La valeur prédite est en accord acceptable avec celle mesurée par Rapp [3] pour le système Ag-In, pour une valeur postulée raisonnable de ce facteur de forme. Pour citer cet article : J.B. Leblond, M. Pignol, D. Huin, C. R. Mécanique? (2013).
\end{abstract}

Key words: Extended Wagner model ; diffusion barriers ; internal oxidation ; external oxidation

Mots-clés : Modèle de Wagner étendu; obstacles à la diffusion; oxydation interne; oxydation externe 


\section{Introduction}

The impact of Wagner's [2] seminal theoretical analysis of internal oxidation of alloys still remains strong in spite of its age. The widely and continuously recognized importance of Wagner's contribution may be appreciated from the numerous works it inspired, including those of Kirkaldy [4], Laflamme and Morral [5], Ohriner and Morral [6], Whittle et al. [7], Christ et al. [8], Stott and Wood [9], Gesmundo and Gleeson [10], Gesmundo et al. [11], Huin et al. [12], Gesmundo et al. [13], Gesmundo et al. [14], Gesmundo and Niu [15], Niu and Gesmundo [16] and Leblond [1], among others. Many of these works were devoted to relaxation of some of the severely restrictive hypotheses originally made by Wagner (consideration of binary alloys only, presence of a single oxidant, oxidation of the sole most reactive metal, formation of a single oxide with a very low solubility product), thus widening the domain of application and increasing the significance of his original work.

In spite of the continued attention paid to Wagner's model, it was not until the very recent work of Leblond [1] that the remark was made that with a slight, natural modification, it can be used to predict the conditions governing the transition from internal to external oxidation in a "self-consistent" way, without introducing any ad hoc parameter. (The use of his model to predict the occurrence of external oxidation was suggested by Wagner [2] himself, but with a postulated, freely adjustable value of the critical volume fraction of oxides leading to the transition). Leblond's [1] suggested modification of the model consisted in considering the diffusion coefficient of oxygen as a decreasing function of the local volume fraction of oxides, so as to account for the possible hindering of inward oxygen diffusion by precipitates acting as obstacles. In spite of the fact that the equations then became nonlinear, Leblond [1] showed that an analytical solution could still be found, with again a uniform fraction of precipitates within the oxidized zone. It was also possible to determine the conditions governing the transition from internal to external oxidation explicitly and analytically. The "critical" volume fraction of precipitates leading to the transition was found to be independent of the diffusion coefficients of elements and be a function of the sole respective specific volumes of the matrix and the oxide.

However the effect of diffusion barriers was modeled, following Kirkaldy's [17] suggestion, by simply reducing diffusion coefficients proportionally to the volume fraction of obstacles. It is well-known from homogenization theories that such a hypothesis only represents an approximation leading to an underestimation of the effect of obstacles; this underestimation may be insignificant or considerable, depending on their volume fraction and shape. As a result, the conditions governing the transition from internal to external oxidation could not be derived accurately: a comparison of the critical fraction of oxides predicted with that actually determined experimentally by Rapp [3] for the silver-indium system thus revealed a discrepancy of a factor of about 2 .

The aim of this work is to pursue Leblond's [1] analysis by using a more accurate formula for the reduction of diffusion coefficients by oxide precipitates acting as barriers. This formula will be established by using a thermal analogy and performing a large number of finite element computations of the overall thermal conductivity of elementary representative cells containing nonconducting inclusions with various volume fractions and shapes. It is hoped that this will lead to transformation of Leblond's [1] essentially qualitative predictions of the onset of external oxidation into fully quantitative ones.

The paper is organized as follows:

- Section 2 presents the numerical simulations performed, and the heuristic formula deduced from there for the overall thermal conductivity of some representative cell containing nonconducting inclusions.

- Section 3 introduces the nonlinear variant of Wagner's model considered, incorporating the formula just derived.

Email addresses: jbl@lmm.jussieu.fr (Jean-Baptiste Leblond), moise.pignol@areva.com (Moïse Pignol), didier.huin@arcelormittal.com (Didier Huin). 
- Section 4 expounds the analytic solution of the equations of the extended model, and derives conditions governing the transition from internal to external oxidation from there.

- Section 5 finally compares the predicted value of the critical fraction of oxides at the onset of external oxidation to that measured by Rapp [3] for the silver-indium system, showing that an acceptable agreement may be reached for a reasonable postulated value of the aspect ratio of oxide precipitates.

\section{Numerical calculation of the overall thermal conductivity of a medium containing non- conducting obstacles}

\subsection{Principle of the simulations}

Several homogenization theories are available to calculate the value of the "homogenized" diffusion coefficient of heterogeneous media. However, none of these theories provides accurate predictions over the full range of possible volume fractions of inclusions. We shall therefore resort to numerical calculations by the finite element method, using the SYSTUS ${ }^{\circledR}$ code developed by ESI-Group.

Exploiting the analogy between the equations of diffusion of atoms and heat, we shall evaluate the reduction of the diffusion coefficient of a diffusing species arising from the presence of impenetrable obstacles by performing calculations of the reduction of the thermal conductivity arising from the presence of nonconducting inclusions.

This reduction does not only depend on the volume fraction of inclusions, but also on their shape. In problems of internal oxidation, the shape of oxide precipitates depends on the surface energy of the matrix/precipitate interface: when it high, oxides tend to minimize it by assuming spherical shapes, whereas when it is low, its influence becomes negligible and oxides tend to freely spread over the surface of the oxidized sheet where oxygen is available, thereby assuming flat shapes. To schematize these different geometric situations, we shall consider oblate spheroidal inclusions with axis of rotational symmetry perpendicular to the surface, and shape characterized by their aspect ratio

$$
W \equiv \frac{\text { Major axis of spheroid }}{\text { Minor axis of spheroid }} \text {. }
$$

The elementary cell considered will be a cube meshed with cubic elements. Different temperatures will be imposed on two opposite faces of this cube, the other faces being thermally insulated. The stationary temperature field will be computed and the overall conduction coefficient deduced from the total heat flux crossing either of the faces where the temperature is prescribed (calculated through numerical integration over this face).

The various inclusions contained within the cell will be assumed to all have the same aspect ratio. For each value of this aspect ratio, their volume fraction will be gradually increased from zero to unity by a step-by-step procedure. At each step of this procedure, the coordinates of the center of some new, additional inclusion will be chosen at random, together with its axes (between specific bounds). This inclusion will be allowed to extend beyond the cell boundaries and/or overlap with previous ones. Elements with centroid lying inside the inclusion will be ascribed a very small thermal conductivity, thereby collectively building an almost nonconducting region. ${ }^{1}$ A stationary thermal calculation will then be performed, before proceeding to the next step.

In practice, the cell is a cube of side $1 \mathrm{~mm}$ meshed with 512,000 trilinear cubic elements ( 80 in each direction) and 531,441 nodes. An arbitrary value of $1 \mathrm{~W} \mathrm{~mm}^{-1}{ }^{\circ} \mathrm{C}^{-1}$ is adopted for the thermal conduc-

1. A strictly zero conductivity is ruled out because it would lead to a singular matrix in the finite element formulation. 
tivity of the matrix, a value $10^{8}$ times lower being ascribed to the inclusions. A temperature difference of $1{ }^{\circ} \mathrm{C}$ is imposed between the lower and upper faces (parallel to the surface of the sheet modeled).

Figure 1 shows two typical geometries obtained at some late stage of the calculations, when the volume fraction of inclusions is high; Figure 1(a) is for $W=1$ (spherical inclusions) and Figure 1(b) for $W=10$ (very flat inclusions). (Note that the axes of rotational symmetry of the spheroidal inclusions are vertical and therefore perpendicular to the surface of the sheet modeled, as desired).

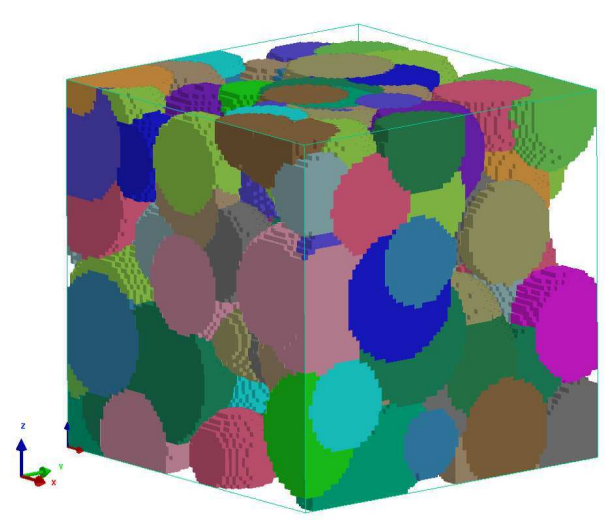

(a) Aspect ratio 1 (spheres)

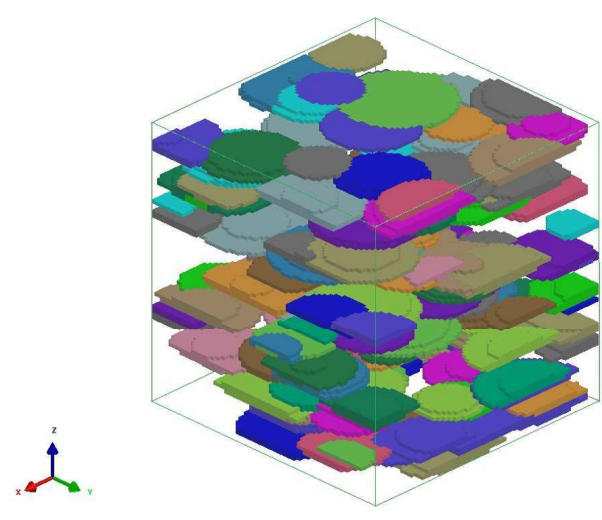

(b) Aspect ratio 10 (very flat spheroids)

Figure 1. Representative cells containing spheroidal obstacles (diffusion in the vertical direction)

\subsection{Results}

Figures 2, 3, 4 and 5 show the results obtained for the values $W=1,2,5,10$ of the aspect ratio of inclusions. The quantities plotted on the horizontal and vertical axes are the volume fraction $F$ of inclusions and the ratio $\lambda^{\mathrm{Hom}} / \lambda$, where $\lambda$ is the thermal conductivity of the inclusion-free matrix and $\lambda^{\text {Hom }}$ the "homogenized" conductivity of the cell. The numbers in the box enclosed in each diagram indicate the minimum and maximum values allowed for the major semi-axis of the inclusions when the random choice is made. In some cases there are several curves for the same minimum and maximum values; they differ because of different random choices of the centers and axes of the inclusions.

Several remarks are in order here:

- For a given value of the aspect ratio of the inclusions, the scatter from one curve to another is relatively small. This permits to define a reasonable "average thermal conductivity" depending only on $F$ and $W$.

- The larger the aspect ratio of the inclusions, the smaller the average conductivity of the cell. Clearly, the flatter the inclusions, the more they hinder diffusion parallel to their axis of rotational symmetry.

- Even in the case of spherical inclusions, for which the average conductivity of the cell is maximal, this conductivity is smaller than what the simple but erroneous "linear rule" $\lambda^{\text {Hom }}=(1-F) \lambda$ would predict; see the dotted line in Figure 2. There is no surprise here since all homogenization theories predict that $\lambda^{\text {Hom }} \leq(1-F) \lambda$, the equality occurring only for very special geometries. 


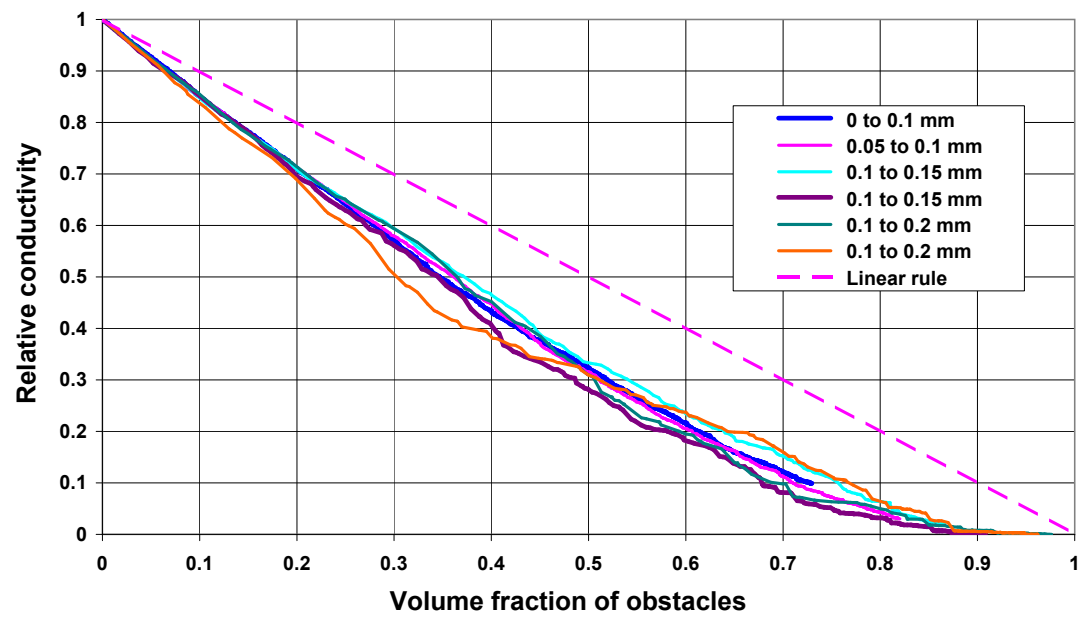

Figure 2. Relative conductivity of a representative cell containing spherical obstacles $(W=1)$

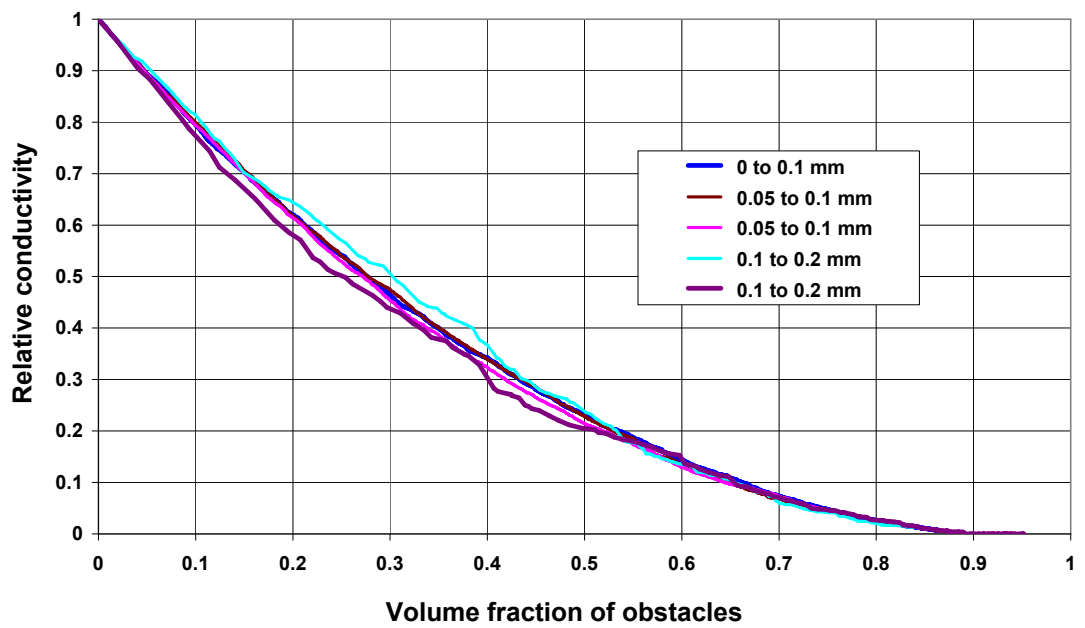

Figure 3. Relative conductivity of a representative cell containing flat spheroidal obstacles with aspect ratio $W=2$

\subsection{Adjustment of numerical results}

Figure 6 compares, for all values of $F$ and $W$ considered, the average thermal conductivity of the cell determined by the finite element method to that given by the approximate heuristic formula

$$
\frac{\lambda^{\mathrm{Hom}}}{\lambda} \simeq(1-F)^{\chi(W)} \quad, \quad \chi(W) \equiv 1+0.55 W .
$$

This formula can be seen to provide a good representation of numerical results, except in some cases for very small values of $\lambda^{\text {Hom }} / \lambda$, which are of little interest here because the transition from internal to external oxidation occurs for larger values. Furthermore, it is sufficiently simple to allow for an analytic prediction of the transition from internal to external oxidation of alloys, as will be seen in the sequel. 


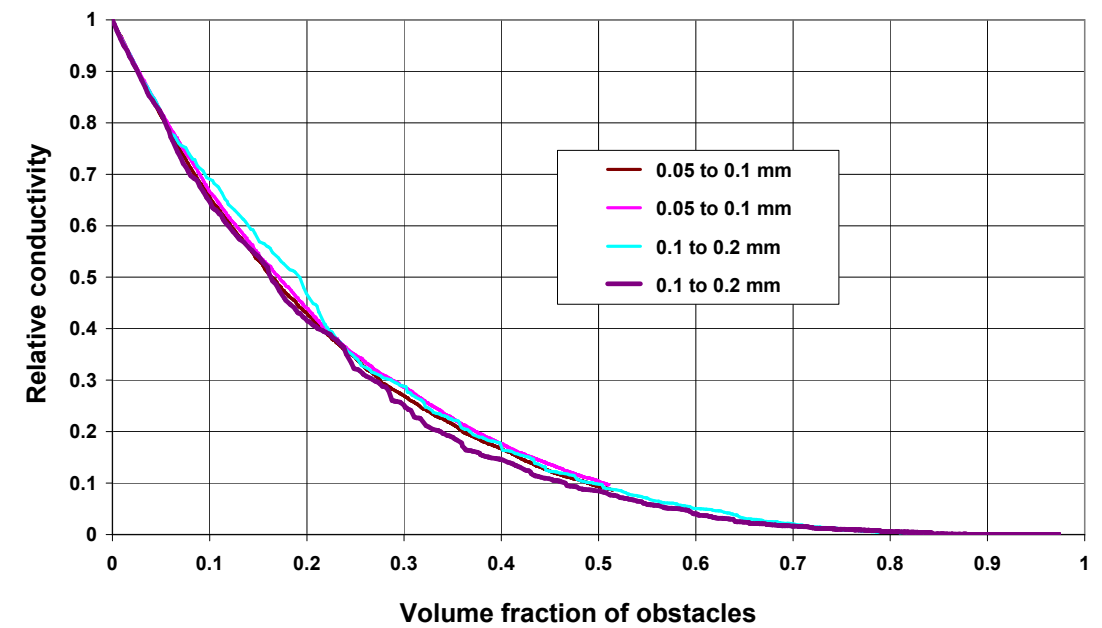

Figure 4. Relative conductivity of a representative cell containing flat spheroidal obstacles with aspect ratio $W=5$

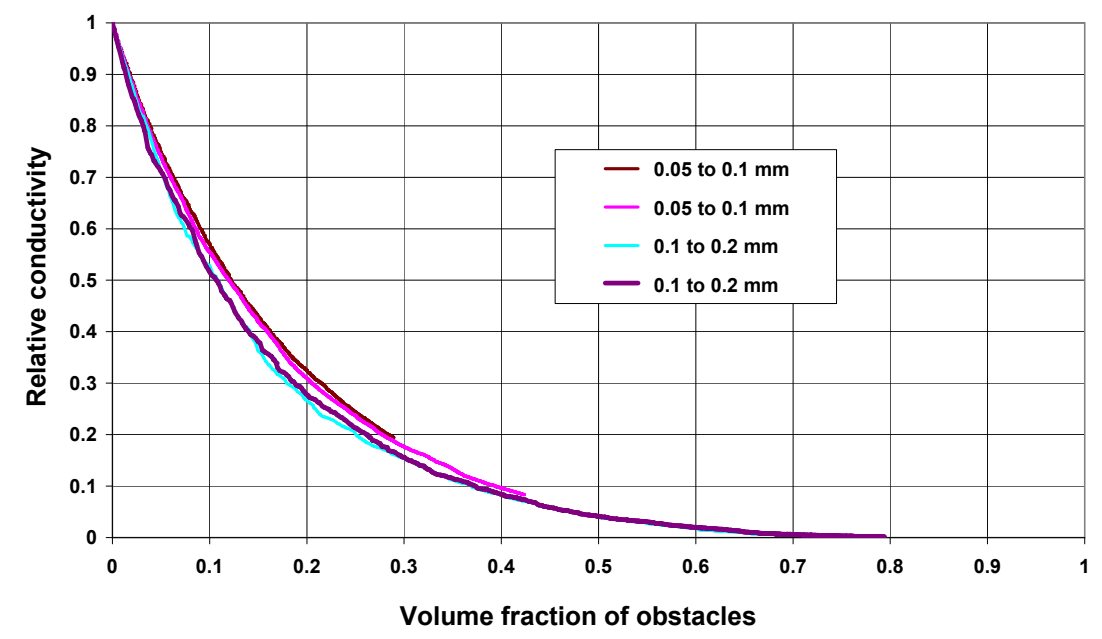

Figure 5. Relative conductivity of a representative cell containing flat spheroidal obstacles with aspect ratio $W=10$

\section{Extended Wagner model accounting for the effect of precipitates as diffusion barriers}

The presentation in this Section is brief since most elements are borrowed from the papers of Wagner [2] and Leblond [1]. Wagner's [2] notations are adopted hereafter except for the use of mass fractions (that is, mass of species per unit mass of the material) instead of molar fractions.

Following Wagner [2], we consider the 1D problem, in the direction $x$, of isothermal internal oxidation of a single element (A) by oxygen $(\mathrm{O})$, resulting in the formation of a single type of oxide with formula $\mathrm{OA}_{\nu}$. The solubility product of this oxide being supposed to be extremely small, the two elements cannot coexist in their dissolved form so that precipitation occurs only on some precipitation front, the position of which is denoted $\xi(t)$. Thus, in the oxidized region $0 \leq x<\xi(t)$, the mass fraction $O$ of dissolved oxygen is nonzero but that $A$ of dissolved oxidizable element is zero, whereas the converse is true in the non-oxidized region $x>\xi(t)$. 


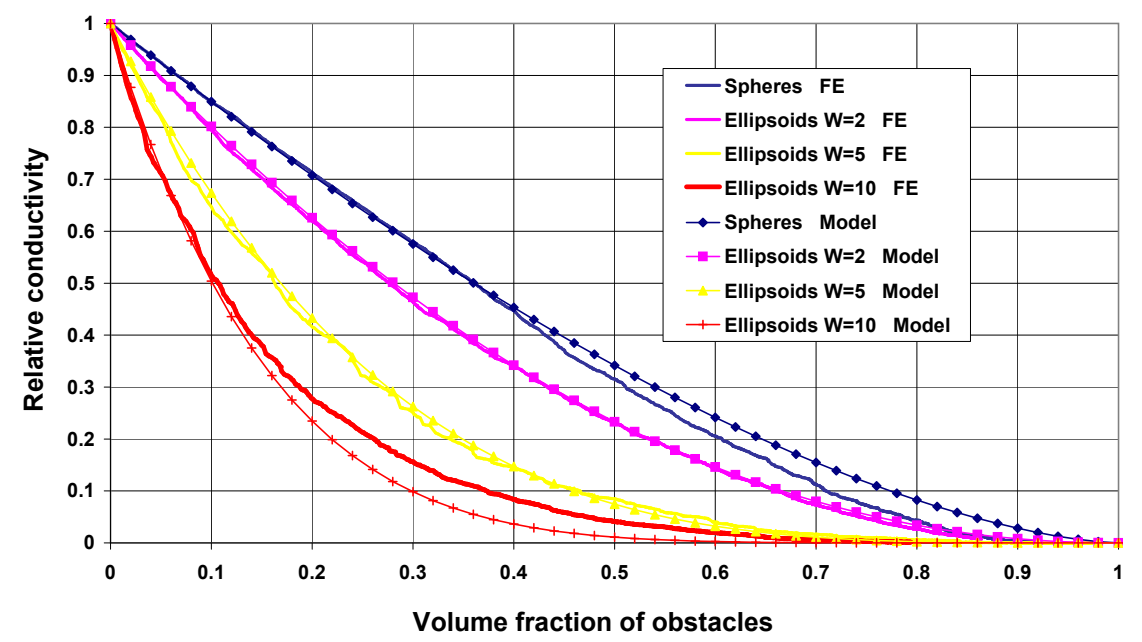

Figure 6. Adjustment of numerical results for the relative conductivity of a representative cell containing obstacles

The equations of the problem read

$$
\begin{cases}\frac{\partial O}{\partial t}(x, t)=\frac{\partial}{\partial x}\left(D_{\mathrm{O}} \frac{\partial O}{\partial x}\right)(x, t) & \text { for } 0<x<\xi(t) \\ \frac{\partial A}{\partial t}(x, t)=\frac{\partial}{\partial x}\left(D_{\mathrm{A}} \frac{\partial A}{\partial x}\right)(x, t) & \text { for } 0<x<\xi(t) \\ O[\xi(t), t]=A[\xi(t), t]=0 & \text { for } t>0 \\ -\nu \frac{D_{\mathrm{O}}}{M_{\mathrm{O}}} \frac{\partial O}{\partial x}\left[\xi(t)^{-}, t\right]=\frac{D_{\mathrm{A}}}{M_{\mathrm{A}}} \frac{\partial A}{\partial x}\left[\xi(t)^{+}, t\right] & \text { for } t>0 \\ O(x, 0)=0 & \text { for } 0<x<+\infty \\ A(x, 0)=A^{\mathrm{c}} & \text { for } 0 \leq x<+\infty \\ O(0, t)=O^{\mathrm{s}} & \text { for } t \geq 0 \\ A(+\infty, t)=A^{\mathrm{c}} & \text { for } t \geq 0 .\end{cases}
$$

Equations $(3)_{1,2}$, where $D_{\mathrm{O}}$ and $D_{\mathrm{A}}$ denote the diffusion coefficients of elements $\mathrm{O}$ and $\mathrm{A}$, are the diffusion equations in the oxidized and non-oxidized regions. Equations (3) $)_{3,4}$, where $M_{\mathrm{O}}$ and $M_{\mathrm{A}}$ denote the molar masses of $\mathrm{O}$ and $\mathrm{A}$, express necessary conditions at the precipitation front; equation $(3)_{4}$ notably expresses the fact that the opposite fluxes of atoms $\mathrm{O}$ and $\mathrm{A}$ at the precipitation front are entirely "consumed" there in the production of $\mathrm{OA}_{\nu}$ molecules. Finally equations $(3)_{5-8}$, where $A^{\mathrm{c}}$ denotes the "core" value of the mass fraction of A defined by the composition of the alloy and $O^{\mathrm{s}}$ the mass fraction of dissolved $\mathrm{O}$ prescribed on the surface, are the initial and boundary conditions.

Wagner's [2] idea was to consider the diffusion coefficients $D_{\mathrm{O}}, D_{\mathrm{A}}$ of elements $\mathrm{O}$ and $\mathrm{A}$ as identical to those, $D_{\mathrm{O}}^{0}, D_{\mathrm{A}}^{0}$, in the non-oxidized matrix. However, in contrast to element A which does diffuse in the non-oxidized region, element $\mathrm{O}$ diffuses in the oxidized one. Hence it is reasonable, assuming that the oxide precipitates act as diffusion barriers and using equation (2), to take $D_{\mathrm{O}}$ and $D_{\mathrm{A}}$ in the following form:

$$
D_{\mathrm{O}} \equiv(1-F)^{\chi(W)} D_{\mathrm{O}}^{0} \quad ; \quad D_{\mathrm{A}} \equiv D_{\mathrm{A}}^{0}
$$

where $F$ denotes the local volume fraction of oxide precipitates and $W$ their aspect ratio. The value of $F$ is to result from the solution of the equations of the model but that of $W$ is assumed to be constant in 
space and time, and given at the present stage of development of the model.

Since mass fractions are used in this work, it is necessary to re-express the local volume fraction $F$ of oxide precipitates in terms of their local mass fraction $P$, as done by Leblond [1]. Consider an elementary domain of material of mass $\delta m$. The volumes occupied by the matrix and the oxide precipitates within this domain are $V_{\mathrm{M}}(1-P) \delta m$ and $V_{\mathrm{P}} P \delta m$ respectively, where $V_{\mathrm{M}}$ and $V_{\mathrm{P}}$ denote the specific volumes (volumes per unit mass) of the two phases. (The slight dependence of $V_{\mathrm{M}}$ upon the dissolved fraction of element A is neglected). Therefore the volume fraction of the precipitates is

$$
F=\frac{V_{\mathrm{P}} P \delta m}{V_{\mathrm{M}}(1-P) \delta m+V_{\mathrm{P}} P \delta m}=\frac{P V_{\mathrm{P}} / V_{\mathrm{M}}}{1-P+P V_{\mathrm{P}} / V_{\mathrm{M}}} .
$$

It follows that the expression $(4)_{1}$ of $D_{\mathrm{O}}$ may be re-written in the form

$$
D_{O}=\frac{D_{O}^{0}}{f(P)} \quad, \quad f(P) \equiv\left(1+\frac{V_{\mathrm{P}}}{V_{\mathrm{M}}} \frac{P}{1-P}\right)^{\chi(W)} .
$$

\section{Analytic solution}

\subsection{Original model}

In a first step, we consider $D_{\mathrm{O}}$ as constant and identical to $D_{\mathrm{O}}^{0}$, and follow Wagner's [2] treatment. The position of the precipitation front being written in the form

$$
\xi(t)=2 \gamma \sqrt{D_{\mathrm{O}} t}
$$

where $\gamma$ is a dimensionless parameter, equations $(3)_{1-3,5-8}$ yield

$$
\left\{\begin{array}{ll}
\frac{O(x, t)}{O^{\mathrm{s}}}=1-\operatorname{erf}\left[x /\left(2 \sqrt{D_{\mathrm{O}} t}\right)\right] / \operatorname{erf} \gamma & \text { for } \quad 0 \leq x \leq \xi(t) \\
\frac{A(x, t)}{A^{\mathrm{c}}}=1-\operatorname{erfc}\left[x /\left(2 \sqrt{D_{\mathrm{A}} t}\right)\right] / \operatorname{erfc}(\gamma \sqrt{\phi}) & \text { for } \quad x \geq \xi(t)
\end{array} \quad \phi \equiv \frac{D_{\mathrm{O}}}{D_{\mathrm{A}}} .\right.
$$

Condition $(3)_{4}$ then provides an equation on $\gamma$ :

$$
\frac{\exp \left(\gamma^{2}\right) \operatorname{erf} \gamma}{\sqrt{\phi} \exp \left(\gamma^{2} \phi\right) \operatorname{erfc}(\gamma \sqrt{\phi})}=\nu \frac{M_{\mathrm{A}}}{M_{\mathrm{O}}} \frac{O^{\mathrm{s}}}{A^{\mathrm{c}}} .
$$

The mass fraction $P$ of oxide precipitates in the oxidized zone may be obtained from the fluxes of elements at the precipitation front; it is independent of $x$ and $t$ and given by

$$
P=A^{\mathrm{c}} \frac{M_{\mathrm{p}}}{\nu M_{\mathrm{A}}} \frac{\exp \left(-\gamma^{2} \phi\right)}{\gamma \sqrt{\pi \phi} \operatorname{erfc}(\gamma \sqrt{\phi})}
$$

where $M_{\mathrm{P}}$ is the molar mass of the molecule $\mathrm{OA}_{\nu}$.

Our interest here is the possible formation of some external scale of oxides, which may occur only if $P$ is comparable to unity. Since $A^{\mathrm{c}}$ is always small in practice, this implies that $P / A^{\mathrm{c}}$ must be large. Now combining equation (9) and the asymptotic expression of the function erfc near infinity, one sees that this cannot be true if $\gamma \sqrt{\phi}$ is of order unity or larger. Therefore we are interested only in the case where $\gamma \sqrt{\phi} \ll 1$, and then $\gamma \ll 1$ also, since $\phi \gg 1$ in practice (oxygen diffuses much more quickly than the oxidizable element). Then equations (8), (9) reduce to

$$
\gamma=\frac{\nu}{2} \sqrt{\pi \phi} \frac{M_{\mathrm{A}}}{M_{\mathrm{O}}} \frac{O^{\mathrm{s}}}{A^{\mathrm{c}}} \quad ; \quad P=\frac{2}{\pi \nu^{2} \phi} \frac{M_{\mathrm{O}} M_{\mathrm{P}}}{M_{\mathrm{A}}^{2}} \frac{\left(A^{\mathrm{c}}\right)^{2}}{O^{\mathrm{s}}} .
$$




\subsection{Extended model}

We now consider the extended model in which $D_{\mathrm{O}}$ and $D_{\mathrm{A}}$ are given by equation (4). Since the expression of $D_{\mathrm{O}}$ makes the equations of the model nonlinear, it seems improbable at first sight that an analytic solution can still be found, but this conclusion reveals wrong upon closer inspection, as was first noted by Leblond [1] for a simpler version of the model.

The key point here is to look, following Leblond [1], for a solution in which the mass fraction $P$ of oxides is still uniform within the oxidized zone. With this hypothesis, equation (5) implies that $D_{\mathrm{O}}$ is also uniform in this zone, so that Wagner's solution still applies and again yields a uniform $P$-value, consistent with the hypothesis made. Thus $P$ is still given by equation $(10)_{2}$, with the sole difference that $\phi$ is no longer now a given constant but a function of $P$ defined by equations $(5)$ and $(7)_{3}$. This provides an equation on $P$ which may be written in the form

$$
f(P)=\alpha P \quad, \quad \alpha \equiv \frac{\pi \nu^{2}}{2} \frac{D_{\mathrm{O}}^{0}}{D_{\mathrm{A}}^{0}} \frac{M_{\mathrm{A}}^{2}}{M_{\mathrm{O}} M_{\mathrm{P}}} \frac{O^{\mathrm{s}}}{\left(A^{\mathrm{c}}\right)^{2}} .
$$

The solutions of equation $(11)_{1}$ are best discussed graphically (Figure 7 ). It is clear from equation $(5)_{2}$ that $f(P)$ is an increasing and convex function of $P$. Therefore equation $(11)_{1}$ may have 0,1 or 2 solutions, depending on the value of the parameter $\alpha$ defined by equation $(11)_{2}$ with respect to some "critical" value $\alpha_{\mathrm{cr}}$.

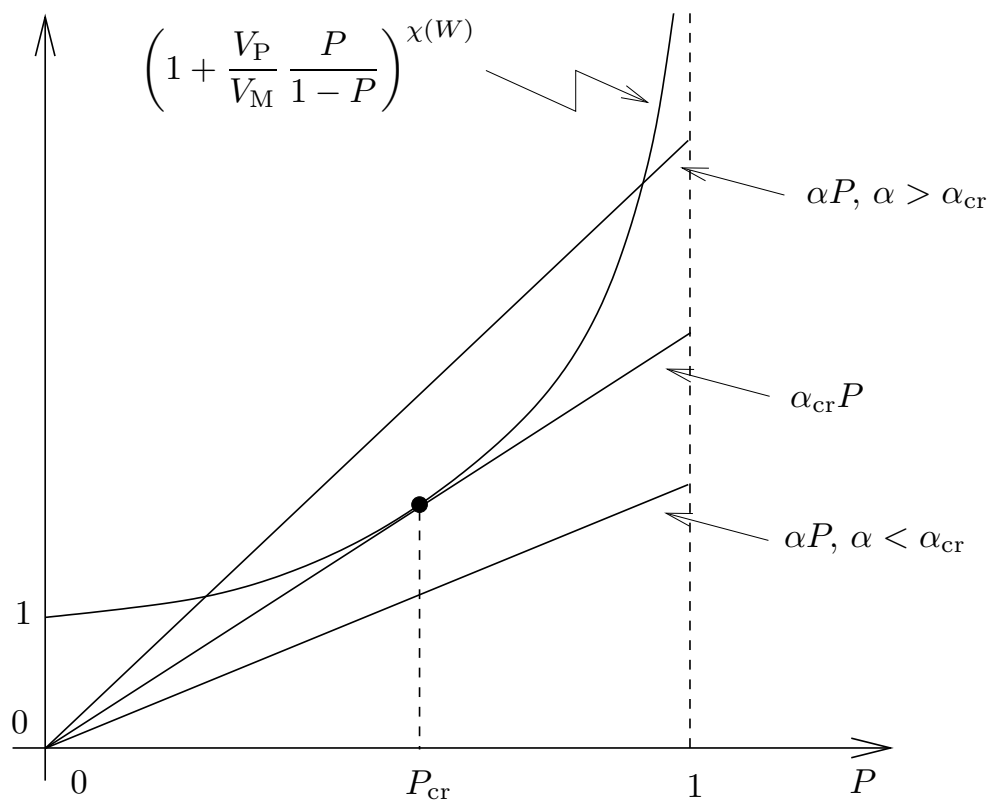

Figure 7. Geometric discussion of the solutions of equation (11) 1

Increase gradually the core value $A^{\mathrm{c}}$ of the mass fraction of the oxidizable element, starting from a zero value for which the mass fraction $P$ of oxides is also nil. The parameter $\alpha$ then gradually decreases from infinity. When $A^{\mathrm{c}}$ is very small, the straight line of slope $\alpha$ is almost vertical so that it cuts the representative curve of the function $f(P)$ near to the origin, thus defining a solution $P$ much smaller than unity. (There is another solution close to unity, but it is inadmissible since it cannot be reached continuously from zero). When $A^{\mathrm{c}}$ increases, the slope $\alpha$ of the straight line decreases so that the abscissa 
$P$ of its first intersection with the curve increases. When $\alpha$ reaches the critical value $\alpha_{\mathrm{cr}}$, the two points of intersection of the straight line and the curve become identical, and $P$ reaches its maximum possible, "critical" value $P_{\mathrm{cr}}$. When $\alpha$ becomes smaller than $\alpha_{\mathrm{cr}}$, no solution with an oxidized zone of finite thickness, as considered until now, can exist any longer. The only possible solution must consist of an infinite concentration of oxides on the surface.

The critical values $\alpha_{\mathrm{cr}}, P_{\mathrm{cr}}$ of the parameters $\alpha$ and $P$ may be calculated analytically. Indeed it follows from the fact that the straight line of slope $\alpha_{\mathrm{cr}}$ is tangent to the curve at the point $P=P_{\mathrm{cr}}$ that

$$
f\left(P_{\mathrm{cr}}\right)=\alpha_{\mathrm{cr}} P_{\mathrm{cr}} \quad ; \quad f^{\prime}\left(P_{\mathrm{cr}}\right)=\alpha_{\mathrm{cr}} .
$$

Eliminating $\alpha_{\mathrm{cr}}$ between these equations, one gets

$$
\frac{f^{\prime}\left(P_{\mathrm{cr}}\right)}{f\left(P_{\mathrm{cr}}\right)}=\frac{1}{P_{\mathrm{cr}}} \Rightarrow \frac{1}{P_{\mathrm{cr}}^{2}}-\frac{2+[\chi(W)-1] V_{\mathrm{P}} / V_{\mathrm{M}}}{P_{\mathrm{cr}}}+1-\frac{V_{P}}{V_{M}}=0
$$

where equation $(5)_{2}$ has been used. The solution of this algebraic equation of the second degree on $1 / P_{\text {cr }}$ is

$$
P_{\mathrm{cr}}=\frac{1}{\frac{\chi(W)-1}{2} \frac{V_{P}}{V_{M}}+1+\sqrt{\left[\frac{\chi(W)-1}{2} \frac{V_{P}}{V_{M}}\right]^{2}+\chi(W) \frac{V_{P}}{V_{M}}}} .
$$

(The other solution is negative, and therefore inadmissible, for $V_{P} / V_{M}>1$, which is always the case in practice). Once the value of $P_{\mathrm{cr}}$ is known, that of $\alpha_{\mathrm{cr}}$ follows from equation $(12)_{1}$ or $(12)_{2}$.

Quite remarkably, the values of both $P_{\mathrm{cr}}$ and $\alpha_{\mathrm{cr}}$ are independent of diffusion coefficients and depend only on the respective specific volumes $V_{M}, V_{P}$ of the matrix and the oxide, plus the shape of the precipitates via the exponent $\chi(W)$.

\section{Discussion and perspectives}

One can find in the literature many experimental values of the critical values (leading to external oxidation) of either the nominal (core) concentration $A^{\mathrm{c}}$ of the oxidizable element for a given value of the superficial concentration of oxygen $O^{\mathrm{s}}$ imposed, or vice-versa; this is quite natural since these quantities are those of most practical interest. However, the critical value of the ratio $O^{\mathrm{s}} /\left(A^{\mathrm{c}}\right)^{2}$ unfortunately depends on the diffusion coefficients of elements through the expression $(11)_{2}$ of the parameter $\alpha$. Since as a rule measurements of these coefficients involve notable experimental errors, direct comparisons between experimental and theoretical critical values of either $A^{\mathrm{c}}$ or $O^{\mathrm{s}}$ would be hampered by important uncertainties.

A comparison of experimental and theoretical values of the critical fraction $P_{\text {cr }}$ of precipitates, free of such uncertainties, is therefore much more preferable. Unfortunately literature does not abound in experimental values of $P_{\text {cr }}$. However a paper of Rapp [3] contains an experimental estimate of the critical volume fraction of $\mathrm{In}_{2} \mathrm{O}_{3}$ for the silver-indium system, amounting to 0.3 . The values of the specific volumes of $\mathrm{Ag}$ and $\mathrm{In}_{2} \mathrm{O}_{3}$ being $V_{\mathrm{M}}=0.095 \mathrm{~cm}^{3} \mathrm{~g}^{-1}$ and $V_{\mathrm{P}}=0.139 \mathrm{~cm}^{3} \mathrm{~g}^{-1}$ respectively, the corresponding critical mass fraction is 0.23 . Now for these specific volumes, the critical mass fraction of the oxide $\operatorname{In}_{2} \mathrm{O}_{3}$ deduced from equation (14) is as follows, for various values of its aspect ratio $W$ :

- for $W=1: P_{\text {cr }}=0.338$;

- for $W=3: P_{\mathrm{cr}}=0.221$;

- for $W=5: P_{\text {cr }}=0.164$.

The value of 0.221 obtained for an aspect ratio of 3 agrees quite well with Rapp's [3] experimental estimation. This aspect ratio is reasonable; it cannot unfortunately be compared to the actual one in Rapp's [3] experiments, which is not provided in his paper. 
This work is liable to at least two extensions:

- As mentioned in the Introduction, Wagner's [2] model involves several severely restrictive hypotheses. Most of these hypotheses can however be removed, provided that the solution is no longer looked after in analytical form but purely numerically; see e.g. the works of Huin et al. [18], Feulvarch et al. [19] and Brunac et al. [20]. The more general models thus defined could be used in conjunction with our expression $(4)_{1}$ of the diffusion coefficient of oxygen in the presence of oxides acting as obstacles, to predict the transition from internal to external oxidation in more complex conditions than those envisaged by Wagner.

- More fundamentally, the aspect ratio of oxides was considered as fixed and given in the present work. In reality, as mentioned in Subsection 2.1, this aspect ratio depends on the surface energy of the matrix/precipitate interface. A more basic development would consist of deriving it in some way from this surface energy, maybe through combined use of some microscopic model of diffusion.

\section{References}

[1] J.B. Leblond, A note on a nonlinear variant of Wagner's model of internal oxidation, Oxid. Metals 75 (2011) $93-101$.

[2] C. Wagner, Reaktionstypen bei der Oxydation von Legierungen, Zeitschrift für Elektrochemie 63 (1959) $772-782$ (in German).

[3] R.A. Rapp, The transition from internal to external oxidation and the formation of interruption bands in silver-indium alloys, Acta Metall. 9 (1961) 730-741.

[4] J.S. Kirkaldy, On the theory of internal oxidation and sulphation of alloys, Can. Metall. Quart. 8 (1969) 35-38.

[5] G. Laflamme, J.E. Morral, Limiting cases of subscale formation, Acta Metall. 26 (1978) 1791-1794.

[6] E.K. Ohriner, J.E. Morral, Precipitate distribution in subscales, Scripta Metall. 13 (1979) 7-10.

[7] D.P. Whittle, F. Gesmundo, B.D. Bastow, G.C. Wood, The formation of solid solution oxides during internal oxidation, Oxid. Metals 16 (1981) 159-174.

[8] H.J. Christ, H.G. Sockel, W. Christl, Carburization of high-temperature materials. I. Mathematical model description of the penetration and simultaneous precipitation of a compound of the diffusing element, Werkstoffe und Korrosion 37 (1986) 385-390.

[9] F.H. Stott, G.C. Wood, Internal oxidation, Mater. Sc. Technol. 4 (1988) 1072-1078.

[10] F. Gesmundo, B. Gleeson, Oxidation of multicomponent two-phase alloys, Oxid. Metals 44 (1995) $211-237$.

[11] F. Gesmundo F., F. Viani, Y. Niu, The internal oxidation of two-phase binary alloys under low oxidant pressure, Oxid. Metals 45 (1996) 51-76.

[12] D. Huin, V. Lanteri, D. Loison, P. Autesserre, H. Gaye, Modelling of internal oxidation of several elements, Microscopy of Oxidation - 3, Newcomb and Little, eds., the Institute of Metals, London, pp. 573-586 (1997).

[13] F. Gesmundo F., F. Viani, Y. Niu, The internal oxidation of two-phase binary alloys beneath an external scale of the less-stable oxide, Oxid. Metals 47 (1997) 355-380.

[14] F. Gesmundo, P. Castello, F. Viani, C. Roos, The effect of supersaturation on the internal oxidation of binary alloys, Oxid. Metals 49 (1998) 237-260.

[15] F. Gesmundo, Y. Niu, The formation of two layers in the internal oxidation of binary alloys by two oxidants in the absence of external scales, Oxid. Metals 51 (1999) 129-158.

[16] Y. Niu, F. Gesmundo, An approximate analysis of the external oxidation of ternary alloys forming insoluble oxides. I: High oxidant pressures, Oxid. Metals 56 (2001) 517-536.

[17] J.S. Kirkaldy, Ternary diffusion and its relationship to oxidation and sulfidation, Oxidation of Metals and Alloys, Douglass, ed., American Society of Metals, Metals Park, pp. 101-114 (1971). 
[18] D. Huin, P. Flauder, J.B. Leblond, Numerical simulation of internal oxidation of steels during annealing treatments, Oxid. Metals 64 (2005) 131-167.

[19] E. Feulvarch, J.M. Bergheau, J.B. Leblond, An implicit finite element algorithm for the simulation of diffusion with phase changes in solids, Int. J. Num. Meth. Engng. 78 (2009) 1492-1512.

[20] J.B. Brunac, D. Huin, J.B. Leblond, Numerical implementation and application of an extended model for diffusion and precipitation of chemical elements in metallic matrices, Oxid. Metals 73 (2010) 565-589. 